ISSN 0103-5150

Fisioter. Mov., Curitiba, v. 24, n. 2, p. 285-298, abr./jun. 2011

Licenciado sob uma Licença Creative Commons

\title{
Proposta de um protocolo de avaliação fisioterapêutica para os pés de diabéticos
}

\author{
Proposal of a protocol physiotherapeutic assessment for diabetics feet
}

\author{
Sarah de Souza Mendonça ${ }^{[a]}$, Juliana de Sant'Anna Morais ${ }^{[\mathrm{b}]}$, \\ Maria Catarina Gomes Gadelha de Moura ${ }^{[c]}$ \\ [a] Fisioterapeuta graduada pela Faculdade Integrada do Recife (FIR), Recife, PE - Brasil, e-mail: ssmendonca@gmail.com \\ [b] Fisioterapeuta graduada pela Faculdade Integrada do Recife (FIR), Recife, PE - Brasil, e-mail: julianafisio.ce@gmail.com \\ [c] Mestre, professora do curso de Fisioterapia da Faculdade Integrada do Recife (FIR), Recife, PE - Brasil, e-mail: \\ catarinagadelha@fir.br
}

\section{Resumo}

Introdução: Em virtude dos altos custos sociais e econômicos decorrentes das complicações do diabetes mellitus (DM) e sabendo-se que uma avaliação clínica minuciosa pode prevenir ou reduzir os impactos desse distúrbio, têm-se cada vez mais a necessidade do desenvolvimento de avaliações específicas. Objetivo: 0 presente trabalho tem como objetivo propor um protocolo de avaliação fisioterapêutica para os pés de diabéticos. Materiais e método: A composição do presente artigo resultou de uma revisão de literatura, que foi realizada a partir dos trabalhos indexados nos periódicos das bases de dados LILACS, SciELO, PubMed e MEDLINE. Os descritores utilizados foram: "diabetes mellitus", "fatores de risco", "pé diabético" e "avaliação". Resultados: 0 protocolo proposto compõe-se de três etapas: a primeira para avaliação dos aspectos sociodemográficos, estilo de vida, histórico da doença, antecedentes de problemas nos pés; a segunda para as condições dermatológicas, circulatórias, neurológicas, funcionais e estruturais; e por último, a avaliação de autocuidado com o pé. Conclusão: A avaliação sugerida pode ser útil para a caracterização minuciosa dos diabéticos, e assim identificar aqueles que apresentam maiores riscos de desenvolverem complicações, bem como pode facilitar a determinação de medidas preventivas e o tratamento adequado.

Palavras-chave: Diabetes mellitus. Pé diabético. Avaliação. 


\section{Abstract}

Introduction: Because of the high social and economic costs caused by complications of diabetes and knowing that a clinical evaluation minute can prevent or reduce the impact of this disorder, have become increasingly need to develop specific assessments. Objective: This study aims to propose an assessment protocol for physical therapy for feet of diabetics. Materials and methods: The composition of this article resulted from a literature review that was conducted a literature search through the journals indexed in the databases LILACS, SciELO, PubMed and MEDLINE. The keywords used were "diabetes mellitus", "risk factors", "diabetic foot" and "assessment". Results: The proposed protocol consists of three steps to assess the socio-demographic, lifestyle, disease history, history of foot problems, the skin conditions, circulatory, neurological, functional and structural and the evaluation of self-care with the feet. Conclusion: The evaluation suggested may be useful for detailed characterization of diabetics, and so identify those who pose a greater risk of developing complications and may facilitate the determination of preventive measures and treatment.

Keywords: Diabetes. Diabetic foot. Assessment.

\section{Introdução}

O aumento do número de indivíduos portadores de diabetes mellitus (DM) nos últimos anos tem preocupado os gestores de sistemas de saúde, pois as complicações clínicas provocadas por tal distúrbio metabólico emergem como uma das maiores ameaças à saúde em todo o mundo, com elevados custos econômicos e sociais $(1,2)$. Por haver evidências $(3$, 4) que demonstram que, por meio de uma avaliação minuciosa e orientação desses indivíduos, pode-se prevenir ou reduzir os impactos do DM, tem-se cada vez mais a necessidade do desenvolvimento de avaliações específicas para os diabéticos.

De acordo com as recomendações da Associação Americana do Diabetes (2), são necessárias a formação e a atuação de uma equipe multiprofissional como condição decisiva para favorecer os cuidados aos portadores do DM, de forma a permitir melhor qualidade de vida para estes indivíduos. Poucos estudos relatam a atuação da Fisioterapia na avaliação preventiva de diabéticos, fato este que pode ser justificado, pois as práticas fisioterapêuticas ainda se centram no processo curativo das enfermidades, apesar de as diretrizes da Fisioterapia determinarem também a atuação desse profissional no âmbito da prevenção (5).

O DM é um distúrbio crônico e complexo, decorrente de alterações na síntese, na secreção ou na ação da insulina, que comprometem o metabolismo das gorduras, proteínas e carboidratos no organismo. Os níveis de glicose no sangue no DM se elevam (hiperglicemia), pois não há uma adequada utilização desta pelas células (6).
Em países de diferentes níveis de desenvolvimento, este distúrbio constitui um importante problema de saúde pública, graças a elevadas prevalência e morbimortalidade (7). No Brasil, um estudo multicêntrico encontrou uma prevalência de 7,6\% de diabéticos, entre 30 e 69 anos de idade; destes, quase metade ainda desconhecia ter a doença, e aproximadamente $25 \%$ dos diabéticos previamente diagnosticados não realizavam qualquer tipo de tratamento (8). Estimativas da Organização Mundial de Saúde (OMS) indicam que o número de pessoas com DM na América Latina alcance, em 2025, a marca dos 64 milhões (9).

As principais responsáveis pela morbidade e mortalidade dos diabéticos são as complicações crônicas, que podem ser decorrentes de alterações na microcirculação, causando retinopatia, nefropatia e neuropatia, ou na macrocirculação, levando à cardiopatia isquêmica, doença cerebrovascular e doença vascular periférica $(3,7)$.

Entre as causas mais frequentes de complicações está o pé diabético, que se3 caracteriza como infecção, ulceração e/ou destruição de tecidos profundos associados a alterações neurológicas, graus diferenciados de doença vascular periférica e deformidades osteoarticulares (10). Ele é responsável por um período de internação 59\% mais prolongado quando comparado aos diabéticos sem tal complicação (11). Estima-se que 15\% dos pacientes com DM desenvolverão pelo menos uma lesão no pé ao longo da vida que poderá resultar em amputações (12).

Atualmente, a literatura (10-14) tem comprovado que o diagnóstico precoce das alterações decorrentes 
do DM, associado ao tratamento oportuno e ao bom cuidado com os pés, pode prevenir complicações. Considerando o prognóstico desta população desenvolver lesões incapacitantes em decorrência do atraso no diagnóstico das complicações associadas ao DM, o presente trabalho tem como objetivo propor um protocolo de avaliação fisioterapêutica para os pés de indivíduos diabéticos.

\section{Materiais e método}

A composição do presente artigo resultou de uma revisão da literatura na qual foi realizada uma pesquisa bibliográfica, a partir dos periódicos indexados nas bases de dados LILACS, SciELO, PubMed, além de livros textos clássicos sobre o tema. Utilizaram-se os seguintes descritores: "diabetes mellitus" (diabetes mellitus), "fatores de risco" (risk factors), "pé diabético" (diabetic foot) e "avaliação" (assessment). Todos eles extraídos dos descritores DeCS/MeSH.

Os critérios de inclusão dos artigos consistiram em estudos que tratassem da avaliação clínica das pessoas com DM, que retratassem métodos utilizados pela Fisioterapia na avaliação do paciente diabético e que tivessem sido publicados nos últimos dez anos na língua portuguesa e inglesa. Após o levantamento bibliográfico, realizou-se um estudo exploratório do material encontrado e, em seguida, efetuou-se uma leitura seletiva.

A partir do material selecionado foi elaborada uma proposta de protocolo fisioterapêutico para avaliação dos aspectos sociodemográficos, estilo de vida, histórico da doença; as condições dermatológicas, circulatórias, neurológicas, funcionais e estruturais; e avaliação quanto ao autocuidado com os pés.

\section{Referencial teórico}

No geral, o DM é assintomático nos estágios iniciais e muitas vezes o diagnóstico é obtido a partir das manifestações das suas complicações (15). 0 risco destas aumenta com o decorrer dos anos e favorece o desenvolvimento de incapacidades. Estas incapacidades podem impedir as pessoas de continuar realizando suas atividades diárias e afastando-as do trabalho, graças às internações prolongadas e recorrentes (12).

\section{Complicações associadas ao desenvolvimento do pé diabético}

As lesões do pé diabético resultam da combinação de dois ou mais fatores de risco que atuam concomitantemente e podem ser desencadeadas tanto por traumas intrínsecos como extrínsecos, associados à neuropatia periférica (NP), à doença vascular periférica (DVP) e à alteração biomecânica $(2,4)$.

A NP crônica associada ao DM caracteriza-se pelo comprometimento das fibras sensitivas, motoras e/ou autonômicas. 0 componente sensitivo acarreta perda gradual da sensibilidade protetora, percepção da pressão plantar, temperatura e propriocepção. Quanto ao componente motor, este se caracteriza por hipotrofia da musculatura intrínseca do pé, que pode desencadear deformidades osteoarticulares e alterações na deambulação. Já o comprometimento autonômico reduz ou suprime a sudorese dos pés, deixando-os secos e predispostos a fissuras, além de desencadear alterações arteriovenosas (13).

A DVP não constitui fator de risco isolado para ulcerações, mas quando associada à neuropatia é uma das principais causas não traumáticas de amputação. Por reduzir o fluxo de nutrientes e oxigênio ao tecido, favorece a emergência de úlceras, já que dificulta a cicatrização, assim como também favorece o surgimento de processos infecciosos, graças ao fato de que condições isquêmicas reduzem a ação da antibioticoterapia $(3,16)$.

Quanto à biomecânica da marcha, qualquer limitação dos movimentos das articulações do pé e tornozelo pode alterá-la, ocasionando passo disfuncional (17). Segundo Sumpio (18), na neuropatia periférica, quando já existem deformidades ósseas desenvolvidas nas cabeças metatarsianas dos pés e no antepé, estas representam áreas de excessiva pressão durante a fase de propulsão do calcâneo e de apoio plantar no ciclo da marcha. Por causa da perda da sensibilidade protetora, o trauma repetitivo causado pela caminhada pode não ser percebido e, como resposta fisiológica natural, acarreta a formação de calos, o que aumentará o risco de ulceração (11).

Entende-se que o risco de ulceração é proporcional ao número de fatores de risco, e que estes aumentam 1,7 vezes em pessoas com diagnóstico de neuropatia periférica, subindo para 12 vezes em pessoas com neuropatia e deformidade do pé e para 36 , naquelas com 
neuropatia, deformidade e amputação prévia, quando comparadas a pessoas sem fatores de risco (18).

\section{Prevenção, fisioterapia e diabetes mellitus}

A prevenção das complicações à saúde do diabético, pode ser realizada a partir de avaliações simples e de baixo custo (19). 0 fisioterapeuta está capacitado a realizar essas avaliações, entretanto, sua prática profissional em ações de prevenção e promoção da saúde ainda é bastante restrita e existem muitas controvérsias sobre seu real papel. A inserção deste profissional em ações de cuidados preventivos é de grande importância para a concretização das diretrizes de uma assistência à saúde realmente integral (20).

Ao se avaliar uma pessoa com DM, enfatiza-se a prevenção das complicações nos pés e o fisioterapeuta deve buscar a influência dos fatores que poderão estar envolvidos direta ou indiretamente na instalação das complicações advindas do DM. Ao considerarmos os danos motores, sensoriais e funcionais acarretados pelo diabetes, uma avaliação específica se torna importante para que a construção do protocolo terapêutico atenda às necessidades específicas de cada indivíduo (21).

\section{Resultados}

0 protocolo sugerido será composto por três etapas: na primeira consistirá em um perfil epidemiológico, na segunda abordar-se-ão os aspectos clínicos e na terceira será realizada uma avaliação do autocuidado com os pés. Para a aplicação do protocolo serão necessários uma ficha de avaliação semiestruturada e outros instrumentos avaliativos, como descrito na Tabela 1.

Tabela 1 - Etapas da proposta do protocolo de avaliação fisioterapêutica para diabéticos

\begin{tabular}{|c|c|c|c|}
\hline & Dados & Itens a pesquisar & Materias utilizados \\
\hline \multirow[t]{4}{*}{ Etapa 1} & Identificação & Nome, sexo, idade, endereço, telefone & Protocolo semiestruturado \\
\hline & Sociodemográficos & $\begin{array}{l}\text { Estado civil, ocupação, escolaridade, } \\
\text { renda familiar }\end{array}$ & Protocolo semiestruturado \\
\hline & Estilo de vida & $\begin{array}{l}\text { Tabagismo, etilismo, prática de } \\
\text { atividade física }\end{array}$ & Protocolo semiestruturado \\
\hline & $\begin{array}{l}\text { Histórico do diabetes e } \\
\text { doenças associadas }\end{array}$ & $\begin{array}{l}\text { Histórico familiar, tempo de diagnóstico, realização } \\
\text { de tratamento e doenças associadas }\end{array}$ & Protocolo semiestruturado \\
\hline \multirow[t]{6}{*}{ Etapa 2} & Exame físico & Altura, peso, IMC & Fita métrica e balança \\
\hline & Condição dermatológica & $\begin{array}{l}\text { Úlcera, queimadura, bolha, rachadura, calosidade, } \\
\text { pele ressecada / descamativa, micose interdigital, } \\
\text { unha espessa / farinácea / onicomicose }\end{array}$ & Protocolo semiestruturado \\
\hline & Condição circulatória & $\begin{array}{l}\text { Sinais e sintomas de comprometimento vascular e } \\
\text { índice tornozelo-braço (ITB) }\end{array}$ & $\begin{array}{l}\text { Protocolo semiestruturado, } \\
\text { esfignomanômetro, Doppler } \\
\text { vascular, gel de contato }\end{array}$ \\
\hline & Condição neurológica & $\begin{array}{l}\text { Sensibilidade de proteção e vibratória, } \\
\text { reflexo aquileu, reflexo patelar }\end{array}$ & $\begin{array}{l}\text { Monofilamento de Semmes-Weinstein } \\
\text { de } 10 \mathrm{~g}(5.07) \text {, diapasão } \\
128 \mathrm{~Hz} \text {, martelo de reflexo }\end{array}$ \\
\hline & Condição funcional & $\begin{array}{l}\text { Função muscular, amplitude articular, } \\
\text { testes funcionais }\end{array}$ & $\begin{array}{l}\text { Protocolo semiestruturado, } \\
\text { goniômetro }\end{array}$ \\
\hline & Condição estrutural & $\begin{array}{l}\text { Alterações ósseas, tipo de pé, pontos } \\
\text { de pressão }\end{array}$ & $\begin{array}{l}\text { Protocolo semiestruturado, } \\
\text { tinta, papel }\end{array}$ \\
\hline Etapa 3 & $\begin{array}{l}\text { Avaliação do } \\
\text { autocuidado }\end{array}$ & $\begin{array}{l}\text { Tipo de calçado, higiene com os pés, } \\
\text { corte das unhas }\end{array}$ & Protocolo semiestruturado \\
\hline
\end{tabular}


Etapa 1

No primeiro estágio de aplicação, o profissional irá interrogar o indivíduo sobre os dados relacionados à sua identificação, aspectos sociodemográficos, estilo de vida, histórico da doença. Para essa etapa será utilizada somente a ficha de avaliação semiestruturada $(22,23)$.

\section{Etapa 2}

A segunda parte contemplará a avaliação clínica, iniciada com a verificação da altura e peso para o cálculo do Índice de Massa Corpórea (IMC). Para este utilizar-se-á a seguinte fórmula: IMC = Peso (kg) / Altura $^{2}$ (m). Serão considerados dentro da normalidade indivíduos com IMC de 18,5 a 24,9 kg/ $\mathrm{m}^{2}$; com sobrepeso de 25,0 a $29,9 \mathrm{~kg} / \mathrm{m}^{2}$; obesos grau I de 30,0 a $34,9 \mathrm{~kg} / \mathrm{m}^{2}$; obesos grau II de 35,0 a $39,9 \mathrm{~kg} / \mathrm{m}^{2}$ e obesos grau III $\geq 40,0 \mathrm{~kg} / \mathrm{m}^{2}$ (24).

Na sequência realizar-se-á a inspeção da pele para verificar a existência de alterações dermatológicas, dentre elas úlcera, queimadura, bolha, rachadura, calosidade, pele ressecada e/ou descamativa, micose interdigital, unha espessa, farinácea e/ou onicomicose (22).

A avaliação circulatória se processará por meio da investigação de sinais e sintomas que estejam relacionados com alteração circulatória e pelo Índice Tornozelo-Braço (ITB). Este será calculado a partir da maior pressão arterial sistólica no tornozelo dividida pela maior pressão arterial sistólica do braço. Para isso, os indivíduos estarão em posição supina com o esfigmomanômetro posicionado na metade do braço, e acima dos maléolos para os membros inferiores. Para facilitar a detecção dos pulos braquiais e pediosos, utilizar-se-á o aparelho de Dopller vascular manual. A DVP será considerada presente quando o ITB for menor a 0,9 , e sem alterações quando for maior ou igual do que 0,9 (1).

Para a detecção das possíveis alterações neurológicas, será testada a sensibilidade protetora e vibratória, segundo as recomendações do Grupo Internacional sobre Pé Diabético (1). Com o indivíduo em posição supina, o monofilamentos de Simmes-Weinstein de $10 \mathrm{~g}$ (5.07) será aplicado no 1으, 3 e 5o pododáctilos e na cabeça dos seus metatarsos. Inicialmente, o monofilamento deverá ser aplicado no cotovelo do paciente, para que ele perceba o estímulo que será testado e, sem que o paciente olhe para a área a ser avaliada, deverá ser feita uma pressão do monofilamento contra o pé, suficiente para que este faça um arco. A aplicação deve ser repetida duas vezes no mesmo local, sendo alternada com, pelo menos, uma aplicação simulada, na qual o monofilamento não é aplicado. Deverão ser feitas três perguntas por local de aplicação. A sensação protetora será considera presente se o paciente responder corretamente a duas das três aplicações (1).

O diapasão de $128 \mathrm{~Hz}$ testará a sensação vibratória, sendo aplicado perpendicularmente sobre a parte dorsal da falange distal do hálux de ambos os pés. Como no teste anterior, inicialmente o instrumento deve ser aplicado no cotovelo do paciente, e o teste também deve ser executado duas vezes no mesmo local, alternando com uma aplicação simulada. A sensação vibratória será considera presente se o paciente responder corretamente duas das três aplicações (1).

O reflexo Aquileu será avaliado com o indivíduo ajoelhado, de forma que seu tornozelo fique livre para que a percussão seja realizada com o martelo neurológico no tendão de Aquiles. Já para o reflexo patelar, o indivíduo deve estar sentado com os joelhos fletidos e os membros inferiores livres. A percussão deverá ser feita no tendão patelar. As respostas aos testes anteriores serão classificadas em arreflexia, hiporreflexia, reflexo normal ou hiperreflexia (25).

$\mathrm{Na}$ avaliação funcional serão realizados testes de função muscular, amplitude articular e testes funcionais em atividades da vida diária. Os testes de função muscular serão baseados nos protocolos estabelecidos por Kendall et al. (26), sendo graduada de 0 a 5 a função muscular. Serão submetidos ao teste os músculos do pé (flexores e extensores dos dedos e hálux, lumbricais e interósseos) e tornozelo (tibial anterior e tríceps sural). As amplitudes de movimentos articulares de tornozelo e pé (flexão plantar, dorsiflexão, inversão e eversão) serão avaliadas com o uso de um goniômetro universal, segundo a metodologia adotada por Marques (27).

A avaliação funcional dos membros inferiores será feita por meio de alguns testes descritos por Palmer e Epler (28), utilizando como escala para a análise dos dados o número de repetições realizadas pelo indivíduo em cada teste. Será pedido ao sujeito que, em pé, realize dorsiflexão, flexão plantar, 
eversão e inversão, usando de uma linguagem acessível. Sentado, o sujeito deverá realizar a flexão dos dedos pegando um algodão com os pododáctilos, bem como sua extensão, ao levantar os dedos do pé do chão. 0 número de repetições será contado durante o período de 30 segundos.

A orientação dada aos indivíduos será a de repetir o movimento a maior quantidade de vezes possível. Para o teste de eversão e inversão do pé, será utilizada a seguinte classificação: nenhuma repetição = não funcional; de 1 a 2 repetições = pouco funcional; de 3 a 4 repetições = razoavelmente funcional; e de 5 a 6 repetições = funcional. Para os demais testes de flexão e extensão de tornozelo e dedos, será utilizada a seguinte escala: nenhuma repetição = não funcional; de 1 a 4 repetições = pouco funcional; de 5 a 9 repetições = razoavelmente funcional; e de 10 a 15 repetições = funcional (28).

As alterações biomecânicas dos pés serão verificadas por meio da inspeção, a fim de detectar modificações ósseas (hálux valgo, dedos em garra, em martelo ou proeminências ósseas), o tipo de pé (normal, cavo ou plano) e se existe alguma alteração na distribuição do peso sobre os pés, evidenciada pela presença de áreas de pressão excessivas (17). Estas poderão ser obtidas a partir da impressão plantar, sendo que para isso os pés dos indivíduos deverão ser marcados com tinta, e observar-se-ão as áreas de pressão a partir da maior intensidade da tinta em uma papeleta (12).

\section{Etapa 3}

A última etapa do protocolo se destina a avaliar o autocuidado dos diabéticos com seus pés, levando em conta os calçados, higiene dos pés e corte das unhas. Considerar-se-ão quatro características na avaliação dos calçados: estilo (modelo), largura, comprimento e material de fabricação. 0 estilo estará apropriado quando for fechado, preferencialmente, protegendo todo o pé; a largura e comprimento devem ter espaço de um centímetro a mais da anatomia do pé; o material confeccionado em couro macio ou lona/algodão. 0 calçado será apropriado quando as quatro características estiveram corretas (14). Com relação ao corte da unhas, será considerado adequado quando estas tiverem na forma reta e sem aprofundamentos nos cantos (1).

\section{Discussão}

A partir da revisão bibliográfica realizada, não foi identificado estudos científicos que incluíssem em um único protocolo todos os itens propostos pelo presente estudo. 0 único artigo selecionado que relacionou de forma direta o DM e avaliação fisioterapêutica diretamente foi uma pesquisa desenvolvida por Sacco et al. (29), na qual foi aplicado um protocolo que se limitava a investigar apenas as alterações neurológicas.

Com relação a alguns dados sociodemográficos descritos na primeira etapa do protocolo, existem controvérsias quanto à incidência no que se refere ao sexo. A Federação Internacional de Diabetes (30) estima para o ano de 2025 um número de mulheres $10 \%$ maior que o de homens acometidos pela doença. Em estudo realizado por Pitta et al. (31) não foi observada variação significante entre os sexos. Nos estudos de Morais et al. (32) e Pace et al. (14) foi observado que o sexo mais acometido é o feminino. Já em outro estudo (7), o sexo mais acometido é o masculino. Portanto, a predominância de um dos sexos ainda não está demonstrada. Por isso, é de importância constar na avaliação o gênero acometido pelo DM.

Com relação à faixa etária, estudos publicados $(23,33)$ revelam que parte predominante da população diabética está entre os 51 e 60 anos, e a incidência dessa patologia aumenta proporcionalmente com a idade. Diante desse dado, é pertinente salientar que quanto mais avançada for a idade, maior serão as alterações fisiológicas e, consequentemente, o aparecimento das complicações advindas do DM (34).

No que diz respeito ao estado civil, Laurindo et al. (35) evidenciaram que o grau de mortalidade é mais frequente em viúvos e solteiros, sendo relativamente mais baixo entre os casados. 0 conhecimento desse dado pelo profissional se torna importante porque indivíduos que não possam contar com a ajuda de um(a) companheiro(a), que o auxilie na terapêutica, precisarão de um acompanhamento mais de perto da equipe de saúde.

Quanto à escolaridade, tanto em estudo nacional (32) como internacional (4), há um maior percentual de indivíduos com baixo grau escolar, constituindo-se em fator agravante para o desencadeamento de complicações crônicas, pela limitação do acesso às informações, graças ao possível comprometimento das habilidades de leitura, escrita 
e compreensão das atividades de educação para o autocuidado preventivo (22). Entretanto, Cosson et al. (36) não encontraram associação entre o grau de escolaridade e as atitudes de controle do DM e cuidados preventivos do pé diabético em nenhuma das fases de seus estudos.

Em seus estudos, Ochoa-Vigo et al. (22) observaram um pequeno número de registros nos prontuários do Programa Saúde da Família sobre a prática do fumo. Porém, o hábito de fumar é considerado como importante fator de risco para DM, pois constitui um agravante para a obliteração das artérias, estando associado com amputação (37). VieiraSantos et al. (33) verificaram uma razão de prevalência de pé diabético de quase duas vezes entre os portadores da doença que faziam uso do tabaco. Por isso é importante sensibilizar nesta população o abandono desta prática como medida de prevenção e estilo de vida saudável.

No que se refere ao consumo de álcool, existe uma probabilidade de quase três vezes daqueles que utilizam álcool de apresentar complicações macrovasculares (33). Além disso, o consumo habitual e prolongado, presente há mais de dez anos, representa uma condição de alto risco para contrair a polineuropatia alcoólica (33). Quando evidenciado o etilismo, o fisioterapeuta, atuando de forma multiprofissional, deverá encaminhar o indivíduo para acompanhamento especializado.

A prática regular de atividades físicas é recomendada pela Associação Americana do Diabetes (2), pois há melhor controle da glicemia, redução dos riscos cardiovasculares, além de ajudar na perda do sobrepeso. Existem fortes evidencias (38) de que treino de resistência combinado com exercícios aeróbicos em indivíduos adultos com diabetes tipo 2 contribui para a redução dos níveis de glicose no sangue.

O tempo da doença maior que dez anos tem se mostrado como importante fator de risco para ocorrência do pé diabético (2). Al-Maskari et al. (4) encontraram evidências que mostram o tempo de diagnóstico da doença (10-12 anos) como um poderoso fator de risco para o desenvolvimento de neuropatias e doenças vasculares. Entretanto, estudos $(7,13)$ relataram maiores percentuais de pé diabético entre os pacientes com tempo de DM inferior a dez anos.

Garber (39) evidenciou que quanto maior o IMC, maior o risco de comorbidades como hipertensão arterial, doenças cardiovasculares e dislipidemia, que pode favorecer o aumento da mortalidade de indivíduos com DM. Assim, avaliações com esses dados podem facilitar o reconhecimento da necessidade de uma reeducação alimentar.

Para a detecção de insuficiência arterial, o presente estudo está propondo o uso do Índice Tornozelo-Braço (ITB), pois é um método não invasivo e já está bem estabelecido na prática médica para determinar o diagnóstico da DVP (40). Em trabalho realizado com portadores de úlcera venosa crônica detectou-se, pelo uso do ITB, uma taxa de $25 \%$ da presença concomitante de DVP (41). Entretanto, o ITB pode dar um falso negativo e, por isso, propõe-se também a investigação de sinais e sintomas que indiquem comprometimento vascular (3).

A força muscular e a mobilidade articular do pé e do tornozelo de indivíduos diabéticos neuropatas foram testadas por Sacco et al. (29) e ficou evidenciado uma diminuição das mesmas. Os músculos intrínsecos do pé, tibial anterior e tríceps sural, e os eversores do pé foram os mais acometidos. Sendo assim, pode-se entender que a avaliação dos itens propostos pelo presente estudo pode contribuir para melhor diagnosticar alterações neurológicas nos diabéticos.

Atualmente, na literatura existem diversos métodos para a avaliação dos comprometimentos neurológicos em indivíduos diabéticos e muitas controvérsias a respeito do diagnóstico de neuropatia diabética (42). Esta, em geral, é avaliada de maneira subjetiva e não padronizada. Em alguns casos, a presença de alterações nos reflexos e sensibilidade pode facilitar o diagnóstico; estes sinais, entretanto, não estão presentes em todos os diabéticos (43). 0 método mais consolidado de avaliação da neuropatia diabética é o uso do monofilamento, e por esse motivo foi o método escolhido pelo presente estudo.

Uma pesquisa (44) na qual utilizaram o mesmo método deste estudo para avaliar a capacidade funcional comprovou uma correlação entre a perda funcional e a neuropatia diabética. Por causa disso, o presente estudo considerou importante a verificação da capacidade funcional como sendo mais um item a ser considerado no diagnóstico da neuropatia diabética.

Vários modelos de aparelhos para a detecção de pontos de pressão têm sido utilizados, porém seu uso significa altos custos. Como alternativa, métodos semiquantitativos também são propostos 
como alternativa para o dia a dia e, dentre eles, destaca-se o Podotrack Footprint System, método simples e barato que quantifica a pressão plantar por meio da observação da intensidade da impressão cinzenta em papel, podendo ser escolhido como substituto do podoscópio. É bom destacar que a literatura nos mostra que o mecanismo para a medida da pressão plantar ainda não está bem definido, havendo necessidade de mais pesquisas a respeito (12). A relação significante entre a pressão anormal do pé e a incidência de ulceração plantar (23) nos serve como parâmetro de risco para desencadear lesões no pé.

As propriedades dos calçados adquirem relevância na função de proteger os pés de agentes lesivos externos, porém podem constituir-se em agentes lesivos, quando extremamente estreitos, ou extremamente folgados (22). Um estudo (23) mostrou uma recorrência de $83 \%$ de úlceras entre pessoas com DM que usaram calçados convencionais e $27 \%$ naquelas com calçados especialmente confeccionados. Uma vez detectada a alteração biomecânica pela presença de calosidades ou deformidades nos pés ou amputação de dedos, calçados terapêuticos devem ser confeccionados sob medida, juntamente com palmilhas que auxiliem a redução e o amortecimento do efeito da tensão repetitiva (45).

Se as condições dermatológicas dos pés estiverem comprometidas, qualquer prejuízo na pele ou nos pés podem progredir até a instalação de uma lesão grave, podendo atingir tecidos profundos (37). Em outro estudo (11) foram identificados como fatores desencadeantes da formação do pé diabético a falta de higiene do pé e o corte de unhas impróprio, com uma taxa de 73,3\% de indivíduos que não possuíam um autocuidado adequado e desenvolveram complicações no pé. Essas condições dermatológicas poderão constituir parâmetros importantes de avaliação para o fisioterapeuta, no processo de orientação ao cuidado, permitindo-lhe determinar as intervenções básicas e planejar as ações educativas, conforme necessidades do indivíduo.

\section{Considerações finais}

A dimensão do impacto socioeconômico causado pelas consequências da DM podem ser evidenciada a partir dos gastos com internações prolongadas e recorrentes, os números elevados de incapacidades físicas e sociais como perda de emprego e produtividade. Por isso é importante concentrar esforços na procura de métodos de praticidade e eficiência de ampliação em programas preventivos.

Uma avaliação criteriosa, acompanhamento regular, conscientização sobre os cuidados com os pés, entre outras medidas, podem reduzir e/ ou prevenir os agravos advindos do DM. Os aspectos coletados por meio da avaliação sugerida pelo presente estudo poderão servir para traçar o perfil do diabético e, assim, evidenciar aqueles que apresentam maiores riscos de desenvolverem complicações e, dessa forma, terem um acompanhamento mais adequado.

Contudo, a aplicabilidade e eficiência do protocolo só poderão ser avaliadas quando este for inserido na prática clínica. A partir disto é que se poderão ser evidenciadas as possíveis mudanças no protocolo sugerido e, assim, oferecer resolutividade quanto às complicações no pé de indivíduos diabéticos.

\section{Referências}

1. Grupo de Trabalho Internacional sobre Pé Diabético. Diretrizes práticas: abordagem e prevenção do pé diabético. Brasília: Secretaria de Estado de Saúde do Distrito Federal; 2001.

2. American Diabetes Association (ADA). Standards of medical care in diabetes-2010. Diabetes Care. 2010;33(suppl 1):11-61.

3. Wu SC, Driver VR, Wrobel JS, Armstro DG. Foot ulcers in the diabetic patient, prevention and treatment. Vasc Health Risk Manag. 2007;3(1):65-76.

4. Al-Maskari F, El-Sadig M. Prevalence of risk factors for diabetic foot complications. BMC Fam Pract. 2007; 8(1):59. doi: 10.1186/1471-2269-8-59.

5. Sampaio RF. Promoção de saúde, prevenção de doenças e incapacidades: a experiência da fisioterapia/ UFMG em uma Unidade Básica de Saúde. Fisioter Mov. 2002;15(1):19-23.

6. Sociedade Brasileira de Diabetes. Consenso brasileiro sobre diabetes: diagnóstico e classificação do diabete mellito e tratamento do diabetes mellito tipo 2. Rio de Janeiro: Diagraphic; 2003. 
7. Brasileiro JL, Oliveira WTP, Monteiro LB, Chen J, Pinho Jr. EL, Molkenthin S, et al. Pé diabético: aspectos clínicos. J Vasc Br. 2005;4(1):11-21.

8. Brasil. Ministério da Saúde. Indicadores de morbidade e fatores de risco. Taxa de prevalência de diabete mellito. [acesso em 22 abr. 2010]. Disponível em: http://tabnet.datasus.gov.br/cgi/idb2001/d10.htm.

9. Washington. Pan-American Health Organization. Prevalence of diabetes among older adults in seven countries of Latin America and the Caribbean (LAC): the health wellbeing and ageing (SABE) project. [acesso em 11 abr. 2010]. Disponível em: http://www.paho. org/common/Display.asp?Lang=E\&Rec ID=6714.

10. Reiber GE. Epidemiologia das úlceras e amputações do pé diabético. In: Bowker JO, Pfeifer MA. Levin e O’Neal o pé diabético. 6a ed. Rio Janeiro: Di-Livros; 2002. p. 13-33.

11. Pedrosa HC, Nery ES, Sena FV, Novaes C, Feldkircher TC, Dias MSO, et al. O desafio do projeto salvando o pé diabético. Terapia em Diabetes. 1998;4(19):1-10.

12. Boulton A, Pedrosa HC. Abordagem diagnóstica, terapêutica e preventiva da neuropatia periférica. In: Vilar L. Endocrinologia clínica. 3a ed. Rio de Janeiro: Guanabara Koogan; 2006.

13. Ochoa-Vigo K, Pace AE. Pé diabético: estratégias para prevenção. Acta Paul Enferm. 2005;18(1):100-9.

14. Pace AE, Foss MC, Ochoa-Vigo K, Hayashida M. Fatores de risco para complicações em extremidades inferiores de pessoas com diabetes mellitus. Rev Bras Enferm. 2002;55(5):514-21.

15. Lopes CF. Projeto de assistência ao pé do paciente portador de diabetes mellito. J Vasc Bras. 2003;2(1): 79-82.

16. Prompers L, Schaper N, Apelqvist J, Edmonds M, Jude E, Mauricio D, et al. Prediction of outcome in individuals with diabetic foot ulcers: focus on the differences between individuals with and without peripheral arterial disease. The Eurodiale Study. Diabetologia. 2008;51(5):747-55.

17. Boike AM, Hall JO. A practical guide for examining and treating the diabetic foot. Cleve Clin J Med. 2002;69(4):342-8.

18. Sumpio B. Foot ulcers. New Engl J Med. 2000;343 (11):787-93.
19. Camdessanche JP, Jousserand G, Ferraud K, Vial C, Petiot $\mathrm{P}$, Honnorat J, et al. The pattern and diagnostic criteria of sensory neuronopathy: a case-control study. Brain. 2009;132(7):1723-33.

20. Barros FBM. O fisioterapeuta na saúde da população: atuação transformadora. Rio de Janeiro: Fisiobrasil; 2002.

21. Lellis VL. Avaliação, prevenção e intervenção no "pé em risco". Diabetes Clín. 2000;4(5):371-75.

22. Ochoa-Vigo K, Torquato MTCG, Silvério IAS, Queiroz FA, De-La-Torre-Ugarte-Guanilo MC, Pace AE. Caracterização de pessoas com diabetes em unidades de atenção primária e secundária em relação a fatores desencadeantes do pé diabético. Acta Paul Enferm. 2006;19(3):296-303.

23. Meijer JWG, Links TP, Smit AJ, Groothoff JW, Eisma, WH. Evaluation of a screening and prevention programme for diabetic foot complications. Prosthet Orthot Int. 2001;25(2):132-8.

24. Ministério da Saúde. Manual de hipertensão arterial e diabetes mellitus. Brasília: Secretaria de Políticas de Saúde, Ministério da Saúde; 2002.

25. Tanenberg RJ, Schumer MP, Greene DA, Pfeifer MA. Problemas neuropáticos das extremidades inferiores dos pacientes diabéticos. In: Bowker JO, Pfeifer MA. Levin O’Neal: o pé diabético. 6a ed. Rio de Janeiro: Di- Livros; 2002. p. 34-65.

26. Kendall FP, McCreary EK, Provance PG. Músculos: provas e funções. 4a ed. São Paulo: Manole; 1995.

27. Marques AP. Manual de goniometria. São Paulo: Manole; 1997.

28. Palmer L, Epler ME. Princípios das técnicas de exame. In: Palmer L, Epler ME. Fundamentos das técnicas de avaliação musculoesquelética. 2a ed. Rio de Janeiro: Guanabara Koogan; 2000. p. 7-33.

29. Sacco ICN, João SMA, Alignani A, Ota DK, Sartor CD, Silveira LT, et al. Implementing a clinical assessment protocol for sensory and skeletal function in diabetic neuropathy patients at a university hospital in Brazil, Sao Paulo. Med J. 2005;123(5):229-33.

30. International Diabetes Federation. Diabetes. One of the most challenging health problems in the 21st century. [acesso em 21 ago. 2006]. Disponível em: http://www.eatlas.idf.org/. 
31. Pitta GBB, Castro AA, Soares AMMN, Maciel CJJ, Silva JDM, Muniz VMT, et al. Perfil dos pacientes portadores de pé diabético atendidos no Hospital Escola José Carneiro e na Unidade de Emergência Armando Lages. J Vasc BR. 2005;4(1):5-10.

32. Morais GFC, Soares MJGO, Costa MML, Santos IBC. 0 diabético diante do tratamento, fatores de risco e complicações crônicas. Rev Enferm UERJ. 2009; 17(2):240-5.

33. Vieira-Santos ICR, Souza WV, Carvalho EF, Medeiros MCW, Nóbrega MGL, Lima PMS. Prevalência de pé diabético e fatores associados nas unidades de saúde da família da cidade do Recife, Pernambuco, Brasil, em 2005. Cad Saúde Pública. 2008;24(12): 2861-70.

34. Lopes FAM, Oliveira FA. Fatores de risco para o desenvolvimento do pé diabético em sujeitos atendidos pelo Programa de Saúde da Família (PSF). [site de internet] 2004. Disponível em: http://www.uftm.edu.br.

35. Laurindo MC, Recco DC, Roberti DB, Rodrigues CDS. Conhecimento das pessoas diabéticas acerca dos cuidados com os pés. Arq Ciênc Saúde. 2005;12(2):80-4.

36. Cosson ICO, Ney-Oliveira F, Adan LF. Avaliação de medidas preventivas do pé diabético em pacientes de Rio Branco, Acre. Arq Bras Endocrinol Metab. 2005; 49(4):548-556.

37. Gambá MA, Gotlieb SLD, Bergamaschi DP, Vianna LAC. Amputações de extremidades inferiores por diabetes mellitus: estudo-caso controle. Rev Saúde Pública. 2004;38(3):399-404.

38. Sigal RJ, Kenny GP, Boule' NG, Wells GA, Prud'homme $D$, Fortier M, et al. Effects of aerobic training, resistance training, or both on glycemic control in type 2 diabetes: a randomized trial. Ann Intern Med. 2007; 147(6):357-69.
39. Garber AJ. The metabolism syndrome. Med Clin North Am. 2004;88(4):837-46.

40. Kawamura T. Índice Tornozelo-Braquial (ITB) Determinado por esfigmomanômetros oscilométricos automáticos. Arq Bras Cardiol. 2008;90(5):322-6.

41. Bergonse FN, Rivitti EA. Avaliação da circulação arterial pela medida do índice tornozelo/braço em doentes de úlcera venosa crônica. An Bras Dermatol. 2006;81(2):131-5.

42. Moreira RO, Castro AP, Papelbaum M, Appolinário JC, Ellinger VCM, Coutinho WF, et al. Tradução para o português e avaliação da confiabilidade de uma escala para diagnóstico da polineuropatia distal diabética. Arq Bras Endocrinol Metab. 2005;49(6):944-50.

43. Souza A, Nery CAS, Marciano LHSC, Garbino JA. Avaliação da neuropatia periférica: correlação entre a sensibilidade cutânea dos pés, achados clínicos e eletroneuromiográficos. Acta Fisiatr. 2005;12(3):87-93.

44. Sacco ICN, Sartor CD, Gomes AA, João SMA, Cronfli R. Avaliação das perdas sensório-motoras do pé e tornozelo decorrentes da neuropatia diabética. Rev Bras Fisioter. 2007;11(1):27-33.

45. Salome GM, Blanes L, Ferreira LM. Capacidade funcional dos pacientes com diabetes mellitus e pé ulcerado. Acta Paul Enferm. 2009;22(4):412-6.

Recebido: 10/12/2010

Received: 12/10/2010

Aprovado: 09/03/2011

Approved: 03/09/2011 


\section{Proposta de protocolo para avaliação fisioterapêutica para os pés de diabéticos}

\section{Etapa 1 - Identificação, dados sociodemográficos, estilo de vida e história clínica}

Data da avaliação:

1.1 Identificação e dados sociodemográficos

Nome: Sexo: Idade:

Endereço:

Telefones: Estado civil:

Escolaridade: Renda familiar:

1.2 Estilo de vida

\begin{tabular}{|c|c|c|c|c|}
\hline & Sim & Não & Tempo & Frequência \\
\hline \multicolumn{5}{|l|}{ Tabagismo } \\
\hline \multicolumn{5}{|l|}{ Etilismo } \\
\hline $\begin{array}{l}\text { Atividade física } \\
\text { Qual? }\end{array}$ & & & & \\
\hline
\end{tabular}

1.3 Histórico do diabetes e doenças associadas

Histórico familiar:

Tipo: Tempo de diagnóstico:

Tipo de tratamento:

Doenças associadas:

\section{Etapa 2 - Avaliação clínica}

\subsection{Avaliação física}

Peso: Altura: IMC (Peso(kg)/Altura ${ }^{2}(\mathrm{~m})$ : Classificação:

\begin{tabular}{lcc}
\hline Classificação & IMC $\left(\mathrm{Kg} / \mathbf{m}^{2}\right)$ & Classe de obesidade \\
\hline Baixo peso & $<18,5$ & \\
Normal & $18,5-24,9$ & \\
Sobrepeso & $25,0-29,9$ & \\
Obesidade & $30,0-34,9$ & Grau I \\
& $35,0-39,9$ & Grau II \\
& $>40,0$ & Grau III \\
\hline
\end{tabular}


Mendonça SS, Morais JS’A, de Moura MCGG.

\subsection{Avaliação dermatológica}

\begin{tabular}{llllll}
\hline & \multicolumn{2}{c}{ Pé direito } & & \multicolumn{2}{c}{ Pé esquerdo } \\
\cline { 2 - 3 } \cline { 5 - 6 } Alterações dermatológicas & Dorso & Planta & & Dorso & Planta \\
\hline Úlceras & & & & & \\
Queimaduras & & & & & \\
Bolhas & & & & \\
Rachaduras \\
Pele ressecada/descamativa
\end{tabular}

\begin{tabular}{l} 
Alterações dermatológicas \\
\hline Calosidades \\
Unha espessa/farinácea/onicomicose \\
Micose interdigital \\
Corte das unhas impróprios \\
Unha encravada \\
Outros
\end{tabular}

\subsection{Avaliação circulatória}

\subsubsection{Sinais e sintomas}

\begin{tabular}{|c|c|c|}
\hline & $S$ & $\mathbf{N}$ \\
\hline \multicolumn{3}{|c|}{ Palidez à elevação da perna } \\
\hline \multicolumn{3}{|c|}{ Claudicação intermitente } \\
\hline \multicolumn{3}{|l|}{ Pele fria/reluzente } \\
\hline Diminuição dos pelos & & \\
\hline
\end{tabular}

\section{Local da dor}

Dor ao repouso

Dor durante a noite

Outros sinais e sintomas:

\subsection{2 Índice tornozelo/braço}

Pressão arterial:

\begin{tabular}{lll}
\hline Pressão arterial sistólica & Direito & Esquerdo \\
\hline Braço & \\
Tornozelo & \\
\hline
\end{tabular}

ITB:

ITB $\geq 0,9-$ normal

ITB $\geq 0,9-$ normal 


\subsection{Avaliação neurológica}

2.4.1 Sensibilidade tátil (monofilamento de $10 \mathrm{~g}$ )

\begin{tabular}{|c|c|c|c|c|c|c|}
\hline \multirow[b]{2}{*}{ Sensibilidade protetora } & \multicolumn{3}{|c|}{ Pé direito } & \multicolumn{3}{|c|}{ Pé esquerdo } \\
\hline & $1^{\mathrm{a}}$ & $2^{a}$ & $3^{a}$ & $1^{\mathrm{a}}$ & $2^{\mathrm{a}}$ & $3^{a}$ \\
\hline \multicolumn{7}{|l|}{$1^{0}$ pododáctilo } \\
\hline \multicolumn{7}{|l|}{$3^{0}$ pododáctilo } \\
\hline \multicolumn{7}{|l|}{$5^{\circ}$ pododáctilo } \\
\hline \multicolumn{7}{|l|}{ Cabeça do $1^{0}$ pododáctilo } \\
\hline \multicolumn{7}{|l|}{ Cabeça do $3^{0}$ pododáctilo } \\
\hline Cabeça do $5^{0}$ pododáctilo & & & & & & \\
\hline
\end{tabular}

P: presente; A: ausente

\subsubsection{Sensibilidade vibratória}

\begin{tabular}{lllllllll}
\hline & \multicolumn{3}{c}{ Pé direito } & & \multicolumn{3}{c}{ Pé esquerdo } \\
\cline { 2 - 3 } \cline { 5 - 7 } & $1^{\text {a }}$ & $2^{\text {a }}$ & $3^{\text {a }}$ & & $1^{\text {a }}$ & $2^{\text {a }}$ & $3^{\text {a }}$ \\
\hline Falange distal hálux & & & & & & \\
\hline
\end{tabular}

P: presente; A: ausente

\subsubsection{Reflexos}

\begin{tabular}{lll}
\hline & Direito & Esquerdo \\
\hline Reflexo aquileu & \\
Reflexo patelar & \\
\hline
\end{tabular}

\section{Possibilidades}

Arreflexia; hiporreflexia; reflexo normal; hiper-reflexia.

\subsection{Avaliação funcional:}

\subsubsection{Goniometria (em graus)}

\begin{tabular}{lll}
\hline & Direito & Esquerdo \\
\hline Dorsiflexão do tornozelo & & \\
Flexão plantar do tornozelo & & \\
Inversão do tornozelo & \\
Eversão do tornozelo & \\
\hline
\end{tabular}

2.5.2 Teste de força muscular do tornozelo e pé (grau de 0 a 5)

\begin{tabular}{|c|c|c|}
\hline & Direito & Esquerdo \\
\hline \multicolumn{3}{|c|}{ Dorsiflexão do tornozelo } \\
\hline \multicolumn{3}{|c|}{ Flexão plantar do tornozelo } \\
\hline \multicolumn{3}{|c|}{ Flexão dos dedos } \\
\hline \multicolumn{3}{|c|}{ Extensão dos dedos } \\
\hline \multicolumn{3}{|c|}{ Inversão do pé } \\
\hline Eversão do pé & & \\
\hline
\end{tabular}




\subsubsection{Teste funcional}

\begin{tabular}{|c|c|c|}
\hline & Número de repetições & Classificação \\
\hline \multicolumn{3}{|c|}{ Dorsiflexão do tornozelo } \\
\hline \multicolumn{3}{|c|}{ Flexão plantar do tornozelo } \\
\hline \multicolumn{3}{|l|}{ Inversão do tornozelo } \\
\hline \multicolumn{3}{|l|}{ Eversão do tornozelo } \\
\hline \multicolumn{3}{|l|}{ Flexão dos dedos } \\
\hline Extensão dos dedos & & \\
\hline
\end{tabular}

\begin{tabular}{lcl}
\hline & Número de repetições & Classificação \\
\hline Dorsiflexão do tornozelo & 0 & Não funcional \\
Flexão plantar do tornozelo & 1 a 4 & Pouco funcional \\
Flexão dos dedos & 5 a 9 & Razoavelmente funcional \\
Extensão dos dedos & 10 a 15 & Funcional \\
\hline & 0 & Não funcional \\
Inversão do tornozelo & 1 a 2 & Pouco funcional \\
Eversão do tornozelo & 3 a 4 & Razoavelmente funcional \\
& 5 a 6 & Funcional \\
\hline
\end{tabular}

\subsection{Avaliação estrutural}

Presença de deformidades:

Tipo de pé (normal, cavo ou plano):

Impressão plantar

Áreas de pressão excessiva: $\quad$ Sim Não

Quais?

\section{Etapa 3 - Avaliação do autocuidado}

\section{Avaliação do calçado:}

Estilo (modelo):

Largura:

Comprimento:

Material de fabricação:

Higiene do pé:

Tipo de corte da unha: 